\title{
Thyrotoxicosis in patients with COVID-19: the THYRCOV study
}

\section{Andrea Lania ${ }^{1,2}$, Maria Teresa Sandri ${ }^{3}$, Miriam Cellini', Marco Mirani', Elisabetta Lavezzi ${ }^{1}$ and Gherardo Mazziotti ${ }^{1,2}$ on behalf of Humanitas COVID-19 Task Force}

${ }^{1}$ Endocrinology, Diabetology and Medical Andrology Unit, Humanitas Clinical and Research Center, IRCCS, Milan, Italy, ${ }^{2}$ Department of Biomedical Sciences, Humanitas University, Milan, Italy, and ${ }^{3}$ Laboratory Medicine, Humanitas Clinical and Research Center, IRCCS, Milan, Italy
Correspondence should be addressed to G Mazziotti Email gherardo.mazziotti@ hunimed.eu

\begin{abstract}
Objective: This study assessed thyroid function in patients affected by the coronavirus disease-19 (COVID-19), based on the hypothesis that the cytokine storm associated with COVID-19 may influence thyroid function and/or the severe acute respiratory syndrome coronavirus 2 (SARS-CoV-2) may directly act on thyroid cells, such as previously demonstrated for SARS-CoV-1 infection.

Design and methods: This single-center study was retrospective and consisted in evaluating thyroid function tests and serum interleukin-6 (IL-6) values in 287 consecutive patients (193 males, median age: 66 years, range: 27-92) hospitalized for COVID-19 in non-intensive care units.

Results: Fifty-eight patients (20.2\%) were found with thyrotoxicosis (overt in 31 cases), 15 (5.2\%) with hypothyroidism (overt in only 2 cases), and 214 (74.6\%) with normal thyroid function. Serum thyrotropin (TSH) values were inversely correlated with age of patients (rho $-0.27 ; P<0.001$ ) and IL-6 (rho $-0.41 ; P<0.001$ ). In the multivariate analysis, thyrotoxicosis resulted to be significantly associated with higher IL-6 (odds ratio: 3.25, 95\% confidence interval: 1.97-5.36; $P<0.001)$, whereas the association with age of patients was lost $(P=0.09)$.

Conclusions: This study provides first evidence that COVID-19 may be associated with high risk of thyrotoxicosis in relationship with systemic immune activation induced by the SARS-CoV-2 infection.
\end{abstract}

\section{Introduction}

The severe acute respiratory syndrome coronavirus 2 (SARS-CoV-2) is a novel enveloped RNA beta-coronavirus responsible for the coronavirus disease-19 (COVID-19) ranging from asymptomatic cases to severe respiratory involvement (1). The first clinical case of COVID-19 in Italy was diagnosed on February 20, 2020; then the infection has rapidly spread involving at this time more than 100000 subjects and configuring our Country as one of the largest and most serious clusters of COVID-19 in the world.

A subgroup of patients with severe COVID-19 might have a cytokine storm characterized by hyperactivity of
European Journal of Endocrinology

(2020) 183, 381-387
Th1/Th17 immune response with increased production of several proinflammatory cytokines, including interleukin 6 (IL-6) and tumor necrosis factor-alfa $(2,3)$. The cytokine pattern observed in patients with COVID-19 is similar to that occurring in some rheumatological diseases or during immunotherapies for cancer (4). Moreover, the cytokine response described in COVID-19 seems to resemble, at least in part, the immune activation that accompanies inflammatory thyroid diseases. Specifically, a hyperactivation of Th1 response in peripheral lymphocytes was described in patients with autoimmune and drug-induced thyroiditis $(5,6,7)$ and an increase in IL-6 was reported in 
the course of destructive thyroiditis $(8,9,10)$. As a matter of fact, alterations of thyroid function and structure were reported in patients affected by SARS-CoV- $1(11,12)$, but the mechanisms were not clarified and it is still unknown whether a similar involvement may occur also during COVID-19, as a consequence of direct or indirect effects of SARS-CoV-2 on the thyroid gland.

In this retrospective study, we aimed to investigate the prevalence and determinants of altered thyroid function in patients hospitalized for COVID-19.

\section{Subjects and methods}

This is a retrospective single-center study performed on 287 consecutive patients hospitalized for COVID-19 at the Humanitas Clinical and Research Center IRCCS, Rozzano-Milan, Italy, in the period between March 1 and April 1, 2020 (Table 1). The inclusion criteria were (i) hospitalization for COVID-19 diagnosed by realtime reverse transcriptase-polymerase chain reaction assay of nasal and pharyngeal swab specimens and or broncoalveolar lavage fluid associated with clinical and radiological signs of pneumonia (1) and (ii) at least one serum thyrotropin (TSH) measurement after COVID19 diagnosis. Exclusion criteria were (i) treatment with L-thyroxine or anti-thyroid drugs before and at the time of TSH evaluation; (ii) treatment with amiodarone; (iii) immunotherapies started before measurement of TSH values; and (iv) requirement of intubation and mechanical respiratory support at the time of TSH evaluation.

The first end-point of the study was the prevalence of low serum TSH values (i.e. values below the reference range in our laboratory) in hospitalized patients with COVID-19. As secondary end-points we explored: (i) the

Table 1 Demographical and clinical data of COVID-19 patients at the study entry.

\begin{tabular}{lc}
\hline$n$ & 287 \\
Age (years) & $66(27-92)$ \\
Sex (F/M) & $94 / 193$ \\
Duration of COVID-19 (days) before & $5(1-15)$ \\
$\quad$ hospitalization & \\
Arterial hypertension on treatment & $142(49.5 \%)$ \\
Diabetes mellitus on treatment & $70(24.4 \%)$ \\
Dyslipidemia on treatment & $63(22.0 \%)$ \\
Active cancer & $9(3.1 \%)$ \\
Chronic obstructive pulmonary disease & $35(12.2 \%)$ \\
Prior coronary artery disease & $41(14.3 \%)$ \\
Prior stroke & $20(7.0 \%)$ \\
Prior venous thromboembolism & $11(3.8 \%)$ \\
\hline
\end{tabular}

Continuous data were presented as median and range. prevalence of overt and subclinical thyroiddysfunction; (ii) the association between TSH and IL-6 values; (iii) the association between TSH values and sex and age of patients; (iv) the association between TSH values and duration of hospitalization and patients' survival; and (v) the clinical presentation and outcome of overt thyrotoxicosis. These end-points were addressed by a retrospective review of laboratory findings and clinical charts of the patients. The patients' survival and duration of hospitalization were calculated excluding from the analysis those patients who were still hospitalized at the time of retrospective evaluation of clinical charts.

In our hospital, measurement of TSH was included in the routine biochemical evaluation of hospitalized patients with COVID-19. In patients with serum TSH values either below or above the reference range, freethyroxine (FT4) and free-triiodothyronine (FT3) were measured for a comprehensive evaluation of thyroid function. In some patients with overt thyrotoxicosis, thyroid ultrasound and measurements of TSH receptor antibodies (TRAb), thyroglobulin antibodies (TgAb) and thyroperoxidase antibodies (TPOAb) were performed. Thyroid function was evaluated on the first day of hospitalization with blood samples drawn in the fasting condition. Over the hours before the hospitalization, all patients had performed thoracic computed tomograpghy (CT) for diagnosis of pneumonia, but iodine contrast was used in only seven cases in whom thromboembolic events were clinically suspected. Before thyroid function evaluation, 127 patients had received thromboprophylaxis with low-molecular-weight heparin. The measurement of thyroid function was performed 9-14 $\mathrm{h}$ after the last administration of heparin and the specimens were analyzed within $8 \mathrm{~h}$ from the blood samples.

The retrospective study was approved by the Ethical Committee of Humanitas Clinical and Research Center, IRCCS and the patients gave their consent to use the clinical and biochemical data for research purposes.

\section{Biochemical assays}

Serum TSH, FT4, FT3 and IL-6 were measured at 08:00 h using chemiluminescent methods on the Beckman Coulter DxI 800 Access ${ }^{\circledR}$ immunoassay system. In our laboratory, the reference ranges of TSH, FT4, FT3 and IL- 6 were 0.34 $4.80 \mathrm{mU} / \mathrm{L}, 7.82-17.29 \mathrm{pmol} / \mathrm{L}, 3.38-6.45 \mathrm{pmol} / \mathrm{L}$ and $<6.4 \mathrm{pg} / \mathrm{mL}$, respectively. TRAb were determined using the TRACE (Time-Resolved Amplified Cryptate Emission) on the Kryptor analyzer and reference range in our laboratory was $<1.8 \mathrm{IU} / \mathrm{L}$. Overt thyrotoxicosis was defined by low

https://eje.bioscientifica.com 
TSH values and serum FT3 and/or FT4 above the reference ranges. Overt hypothyroidism was defined by high TSH values and serum FT4 and/or FT3 below the reference ranges. Subclinical thyroid dysfunction was defined when TSH was either low or high accompanied by FT4 and FT3 in the reference ranges.

\section{Statistical analysis}

Data were presented as median and range, unless otherwise stated. Based on TSH values, patients were stratified in four groups $(<0.10,0.10-0.33,0.34-4.80$, and $>4.80 \mathrm{mU} / \mathrm{L})$. The comparisons were performed by Mann-Whitney's and Kruskal-Wallis' tests. Frequencies were compared by the Chi-square's test, with Fisher correction when appropriate. Correlations between continuous variables were sought by calculating Spearman's coefficient. A logistic regression analysis was performed and the odds ratio (OR) with 95\% confidence interval (CI) were calculated to evaluate the association between thyrotoxicosis, age of patients and IL-6 values stratified in tertiles. A $P$ value $<0.05$ was considered significant.

\section{Results}

Fifty-eight patients (20.2\%) were found with TSH below the reference range $(<0.10 \mathrm{mU} / \mathrm{L}$ in 16 cases, between 0.10 and $0.33 \mathrm{mU} / \mathrm{L}$ in 42 cases), whereas TSH was normal and high in the remaining 214 (74.6\%) and 15 (5.2\%) patients, respectively. In 214 patients with TSH values in the reference range, 99 (46.3\%) had values between 0.34 and $1.0 \mathrm{mU} / \mathrm{L}$.

Thyroid hormones were measured in 73 patients with abnormal TSH values. Overt thyroid dysfunction was diagnosed in 33 patients (31 with thyrotoxicosis and 2 with hypothyroidism), whereas a subclinical thyroid dysfunction was present in 40 patients (27 with thyrotoxicosis and 13 with hypothyroidism). Before assessment of thyroid function, 30 patients with thyrotoxicosis $(51.7 \%)$ received thromboprophylaxis with low-molecular-weight heparin $(P=0.39$ vs patients without thyrotoxicosis) and 3 patients (5.2\%) had performed thoracic CT with iodine contrast $(P=0.17$ vs patients without thyrotoxicosis).

Patients with overt thyrotoxicosis showed higher serum FT4 values as compared to those with subclinical thyrotoxicosis (22.31 pmol/L, range: $17.38-36.33$ vs 15.10 pmol/L, range: 9.68-16.90; $P<0.001)$, without significant difference in serum FT3 values (4.42 pmol/L, range: 3.49-5.28 vs $4.11 \mathrm{pmol} / \mathrm{L}$, range: $3.00-5.20 ; P=0.195)$. Serum TRAb, TgAb and TPOAb were measured in nine patients and in all of them, they resulted negative. Among the eight patients with overt thyrotoxicosis in whom thyroid ultrasound was performed, signs of thyroid inflammation were reported in two cases, small thyroid nodules in other three patients, whereas the remaining three patients did not show any significant alteration of thyroid structure. Overt thyrotoxicosis was accompanied by atrial fibrillation with high heart rate in ten patients (32.3\%). Moreover, five patients with overt thyrotoxicosis developed thromboembolic events (ischemic stroke in two cases, venous thromboembolism in three cases). No local signs or symptoms of subacute thyroiditis were recorded by the physicians.

Of the patients with overt thyrotoxicosis, 4 were treated with thiamazole (5 $\mathrm{mg}$ per day in all cases), whereas the remaining 27 patients did not receive thyroid-targeting drugs. One patient was withdrawn from thiamazole after 3 days due to increase in liver enzyme. A short-term follow-up was available in seven patients (two treated with thiamazole and five without treatment) showing a reduction in serum FT4 values regardless of the treatment of thyrotoxicosis (Fig. 1).

No significant difference in serum TSH values was found between males and females $(0.83 \mathrm{IU} / \mathrm{L}$, range: $0.01-$ 17.54 vs $1.15 \mathrm{IU} / \mathrm{L}$, range: $0.01-75.40 ; P=0.08$ ).

Serum TSH values were inversely correlated with age of patients (rho: $-0.27 ; P<0.001$ ). Patients with TSH below $0.34 \mathrm{mU} / \mathrm{L}$ were significantly older as compared to patients with higher TSH, without significant difference between $<0.10$ and $0.10-0.33 \mathrm{mU} / \mathrm{L}$ (Fig. 2). Serum IL-6 was measured in 210 patients and resulted to be significantly correlated with TSH values (rho: $-0.41 ; P<0.001)$ and age of patients (rho 0.39; $P<0.001$ ). Serum IL-6 values were significantly higher in patients with TSH $<0.34 \mathrm{mU} / \mathrm{L}$ as compared to those with higher TSH values, without significant difference between $<0.10$ and $0.1-0.33 \mathrm{mU} / \mathrm{L}$ (Fig. 3). No significant differences in age $(P=0.22)$ and IL-6 $(P=0.45)$ were found between subclinical and overt thyrotoxicosis.

In the multivariate logistic regression analysis, thyrotoxicosis resulted to be significantly associated with higher IL-6 (OR: 3.25, 95\% CI: 1.97-5.36; $P<0.001$ ) but not with the age of patients (OR: $1.03,95 \% \mathrm{CI}$ : 0.99-1.06; $P=0.09$ ).

We recorded a total of 60 in-hospital deaths, corresponding to an in-hospital mortality rate of $21.4 \%$ among closed cases $(n=280)$. The in-hospital mortality 


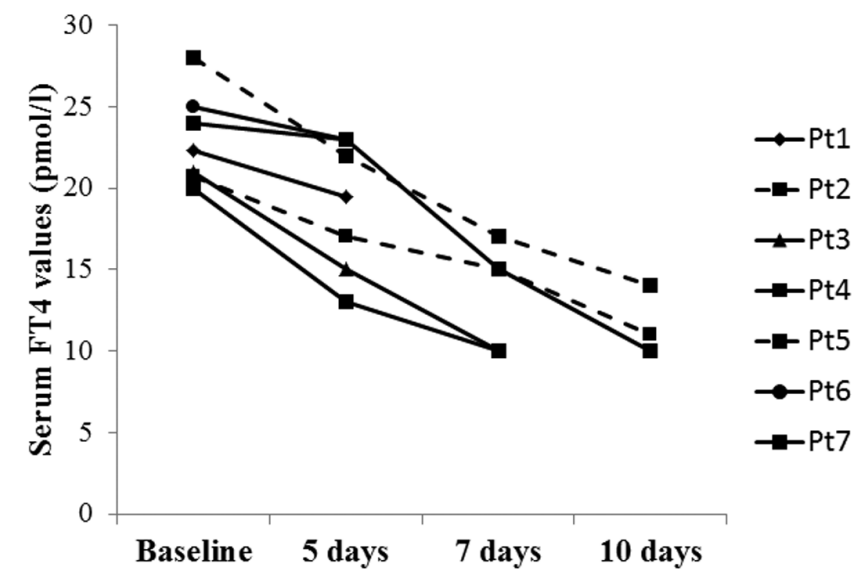

Figure 1

Individual outcomes of serum-free thyroxine (FT4) values in seven patients with overt thyrotoxicosis in the course of COVID-19 who were sequentially evaluated for thyroid function. Two patients (dashed line) were treated with thiamazole, whereas the other five patients (solid line) were not treated with thyroid-targeting drugs.

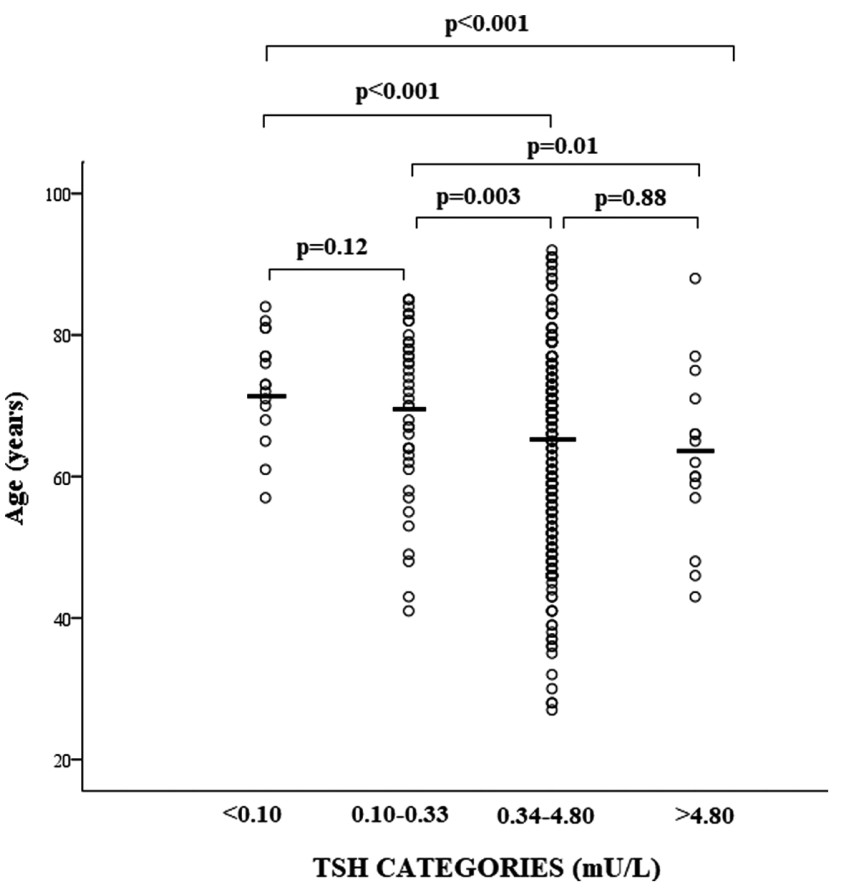

Figure 2

Scatter plot of age in COVID-19 patients stratified for serum thyrotropin (TSH) values. The solid lines identified the median values in each group. Comparisons were performed by Kruskal-Wallis' and Mann-Whitney's tests.

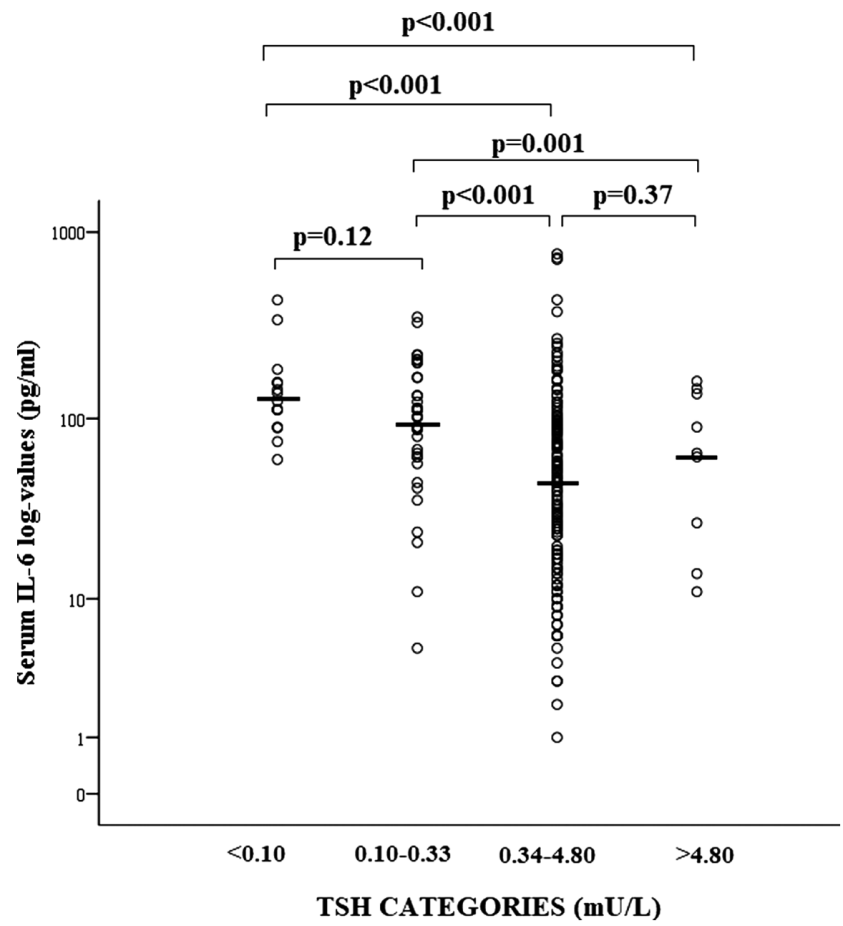

Figure 3

Scatter plot of serum interleukin-6 (IL-6) values expressed in log-scale in COVID-19 patients stratified for serum thyrotropin (TSH) values. The solid lines identified the median values in each group. Comparisons were performed by Kruskal-Wallis' and Mann-Whitney's tests.

rate was significantly lower in patients with TSH values in the reference range as compared to those with either thyrotoxicosis or hypothyroidism (Fig. 4). In discharged patients, the duration of hospitalization resulted to be significantly longer in cases with thyrotoxicosis as compared to those with either normal TSH or hypothyroidism (Fig. 5).

\section{Discussion}

This retrospective study reported, for the first time, high prevalence of overt and subclinical thyrotoxicosis in patients with COVID-19, in close relationship with high circulating levels of IL-6. Noteworthy, the prevalence of hypothyroidism was low in this clinical setting with most of the cases being mild.

The cost-effectiveness and diagnostic value of serum TSH measurement in hospitalized patients are controversial $(13,14,15)$. As a matter of fact, an important derangement of hypothalamic-pituitary-thyroid axis takes place during critical illness, configuring the so-called non-thyroidal 


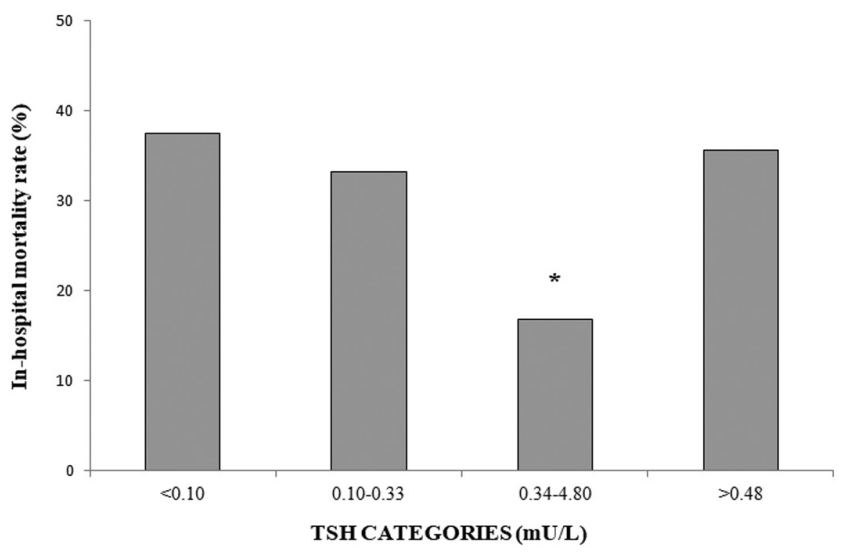

Figure 4

In-hospital mortality rate in COVID-19 patients stratified for serum thyrotropin (TSH) values. $* P<0.05$ vs the other groups.

illness' (NTI) which is consistently characterized by low FT3 values consequent to increased deiodination of T4 in reverse-T3 (16). With increasing severity of illness, low total and FT4, and sometimes low TSH, can be observed. A remarkable number of our hospitalized patients had suppressed TSH values and this finding was very likely expression of thyrotoxicosis rather than NTI. In fact, none of these patients had low FT3 values and in more than one-half of them, FT4 values were above the reference range. Nevertheless, we cannot exclude that a possible coexistent NTI may have influenced the biochemical presentation of thyrotoxicosis, since serum FT3 increased less than FT4, no significant difference in FT3 values was found between overt and subclinical thyroid dysfunction and the recovery of thyrotoxicosis (i.e. in the few patients in whom longitudinal measurements of thyroid hormones were performed) was apparently faster than that observed in non-hospitalized patients. In this context, the role of IL-6 may be hypothesized. In cell cultures, IL- 6 was shown to decrease $\mathrm{T} 3$ secretion possibly contributing to the normal levels of FT3 in our thyrotoxicotic patients (10).

Thyrotoxicosis was found in about $20 \%$ of our patients and several others showed serum TSH in the lower part of the reference range. These findings suggest that COVID-19 may favor the development of thyrotoxicosis at a higher incidence than that expected in the general population (17). Although our study was not designed to clarify the mechanisms responsible for thyroid dysfunction in patients with COVID-19, it is reasonable to hypothesize that thyrotoxicosis was caused by destructive thyroiditis. This hypothesis was supported by the findings that thyrotoxicosis was often mild and improved spontaneously during follow-up . A further finding supporting the

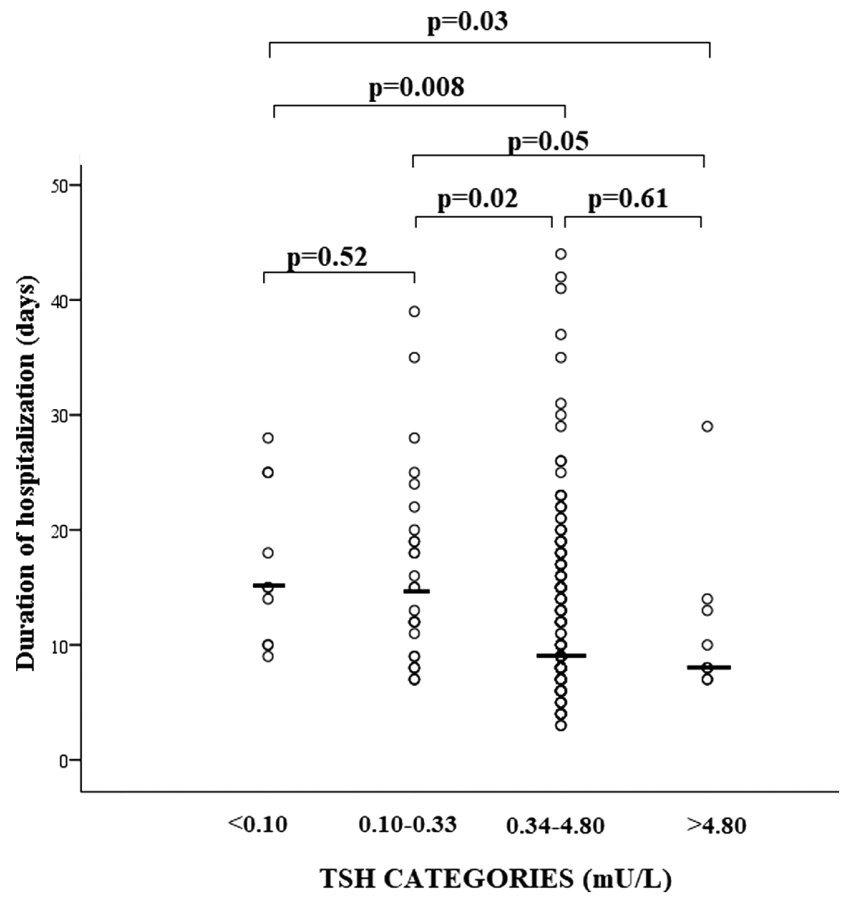

Figure 5

Scatter plot of hospitalization length in COVID-19 patients stratified for serum thyrotropin (TSH) values. The solid lines identified the median values in each group. Comparisons were performed by Kruskal-Wallis' and Mann-Whitney's tests.

diagnosis of destructive thyroiditis was the negativity for TRAb in the few patients in whom these antibodies were evaluated, although the absence of circulating TRAb cannot exclude Graves-like mild hyperthyroidism in all cases (18). The close relationship between thyrotoxicosis and higher serum IL- 6 in our patients suggests that thyroid gland inflammation might be triggered and sustained by the cytokine storm associated with COVID-19, mimicking thyroid disorders developing during the course of immunotherapies $(19,20)$. An alternative hypothesis is the possible direct action of SARS-CoV-2 on thyroid gland (12), based on the evidence that several tissues and organs may be directly damaged by the virus during COVID-19 (21). Indeed, there is evidence that thyroid tissue highly expresses the angiotensin-converting enzyme 2 (22), which is the protein used by SARS-CoV-2 for invading human cells (23).

Besides the pathophysiological aspects, this study highlights some specific clinical concerning the clinical relevance and management of thyrotoxicosis in patients of COVID-19. Sixteen percent of patients with overt thyrotoxicosis developed thromboembolic events, a rate being more than two times higher than that recently 
reported in COVID-19 patients hospitalized in nonintensive care units (24). This finding along with the high prevalence of atrial fibrillation and the close relationship between suppressed TSH and high mortality rate and longer hospitalization suggest that thyrotoxicosis may be clinically relevant in COVID-19 patients.

As patients were isolated, thyroid scintigraphy was not allowed and ultrasound evaluation of thyroid gland was performed only in few cases. Therefore, the therapeutic approach in our COVID-19 patients was empiric and rather limited. Thiamazole was used in very few patients based on the hypothesis that thyrotoxicosis was caused by destructive thyroiditis in which no specific therapy is required beyond symptomatic treatment when indicated. There was likely a rationale for using glucocorticoids in order to accelerate the resolution of thyroid dysfunction (20). However, these drugs were not used due to concerns about potential impairment of underlying SARS-CoV-2 infection during corticosteroid therapy (25). Therefore, most of our patients were followed-up without specific treatment of thyrotoxicosis and thyroid function improved spontaneously. One could argue that this approach may be not completely safe in the setting of COVID-19, since thyrotoxicosis might increase the cardiovascular risk even after short exposure to thyroid hormone excess (26) favoring the cardiovascular complications and arrhythmias described in patients with SARS-CoV-2 infection $(1,27,28)$. Noteworthy, thyrotoxicosis may favor the development of fatal arrhythmias in the presence of prolonged QT interval (29), a common event in COVID19 , as an effect of therapies and/or electrolyte disorders (1, 28 ). On the other hand, the prevention and treatment of thyrotoxicosis-related heart disorders may be challenging in the setting of COVID-19, since some drugs used to counteract the negative effects of thyroid hormones on heart (e.g. beta-blockers) should be administered with caution in patients with pre-existing alterations of heart rhythm (28).

This study has limitations. The lack of an independent cohort of patients with pneumonia did not allow to define the true impact of COVID-19 on thyroid function and the risk of thyrotoxicosis in this clinical setting. In several patients, thyroid function was assessed in the course of treatment with low-molecular-weight heparin for prevention of venous and arterial thromboembolic complications of COVID-19 (24). One could argue that the use of heparin may have favored the elevation of FT4 in our patients as an effect of displacement of the thyroid hormone from the binding proteins (30). However, the suppressed TSH values associated with high FT4 were highly suggestive for true thyrotoxicosis rather than a drug interference. Moreover, it is reasonable to hypothesize that the assay interference induced by low-molecularweight heparin was likely marginal, since several hours elapsed between the biochemical evaluation of thyroid function and the last administration of heparin and the hormone assays were performed in few hours after blood samples (30). The retrospective design along with the relatively small size of the study group, precluded to investigate the independent impact of thyrotoxicosis on the outcome of COVID-19. Moreover, it was not allowed to investigate whether thyroid function might be influenced by immunotherapies used in COVID-19 $(20,31)$. Furthermore, this study did not clarify whether chronic thyroiditis may develop after the resolution of COVID-19 (32).

In conclusion, this retrospective study provides first evidence of high risk of thyrotoxicosis after SARS-CoV-2 infection. Future prospective studies will clarify the clinical and prognostic relevance of thyrotoxicosis in patients with COVID-19.

\section{Declaration of interest}

The authors declare that there is no conflict of interest that could be perceived as prejudicing the impartiality of this study.

\section{Funding}

This research did not receive any specific grant from any funding agency in the public, commercial or not-for-profit sector.

\section{References}

1 Guan WJ, Ni ZY, Hu Y, Liang WH, Ou CQ, He JX, Liu L, Shan H, Lei C, Hui DSC et al. Clinical characteristics of coronavirus disease 2019 in China. New England Journal of Medicine 2020382 1708-1720. (https://doi.org/10.1056/NEJMoa2002032)

2 Wu D \& Yang XO. TH17 responses in cytokine storm of COVID19: an emerging target of JAK2 inhibitor Fedratinib. Journal of Microbiology, Immunology, and Infection 202053 368-370. (https://doi. org/10.1016/j.jmii.2020.03.005)

3 Prompetchara E, Ketloy C \& Palaga T. Immune responses in COVID19 and potential vaccines: lessons learned from SARS and MERS epidemic. Asian Pacific Journal of Allergy and Immunology 202038 1-9. (https://doi.org/10.12932/AP-200220-0772)

4 Zhang W, Zhao Y, Zhang F, Wang Q, Li T, Liu Z, Wang J, Qin Y, Zhang X, Yan X et al. The use of anti-inflammatory drugs in the treatment of people with severe coronavirus disease 2019 (COVID19): the perspectives of clinical immunologists from China. Clinical Immunology 2020214 108393. (https://doi.org/10.1016/j. clim.2020.108393)

5 Mazziotti G, Sorvillo F, Naclerio C, Farzati A, Cioffi M, Perna R, Valentini G, Farzati B, Amato G \& Carella C. Type-1 response in peripheral CD4+ and CD8+ T cells from patients with Hashimoto's thyroiditis. European Journal of Endocrinology 2003148 383-388. (https://doi.org/10.1530/eje.0.1480383) 
6 Mazziotti G, Sorvillo F, Piscopo M, Morisco F, Cioffi M, Stornaiuolo G, Gaeta GB, Molinari AM, Lazarus JH, Amato G et al. Innate and acquired immune system in patients developing interferon-alpha-related autoimmune thyroiditis: a prospective study. Journal of Clinical Endocrinology and Metabolism 200590 4138-4144. (https://doi.org/10.1210/jc.2005-0093)

7 Farzati B, Mazziotti G, Cuomo G, Ressa M, Sorvillo F, Amato G, La Montagna G, Carella C \& Valentini G. Hashimoto's thyroiditis is associated with peripheral lymphocyte activation in patients with systemic sclerosis. Clinical and Experimental Rheumatology 200523 43-49.

8 Kristensen B, Hegedus L, Madsen HO, Smith TJ \& Nielsen CH. Altered balance between self-reactive Thelper (Th) 17 cells and Th10 cells and between full-length forkhead box protein 3 (FoxP3) and FoxP3 splice variants in Hashimoto's thyroiditis. Clinical and Experimental Immunology 2015180 58-69. (https://doi.org/10.1111/ cei.12557)

9 Trovato M, Ruggeri RM, Sciacchitano S, Vicchio TM, Picerno I, Pellicano G, Valenti A \& Visalli G. Serum interleukin-6 levels are increased in HIV-infected patients that develop autoimmune disease during long-term follow-up. Immunobiology 2018223 264-268. (https://doi.org/10.1016/j.imbio.2017.10.039)

10 Bartalena L, Brogioni S, Grasso L \& Martino E. Interleukin-6 and the thyroid. European Journal of Endocrinology 1995132 386-393. (https:// doi.org/10.1530/eje.0.1320386)

11 Leow MK, Kwek DS, Ng AW, Ong KC, Kaw GJ \& Lee LS. Hypocortisolism in survivors of severe acute respiratory syndrome (SARS). Clinical Endocrinology 200563 197-202. (https://doi. org/10.1111/j.1365-2265.2005.02325.x)

12 Wei L, Sun S, Xu CH, Zhang J, Xu Y, Zhu H, Peh SC, Korteweg C, McNutt MA \& Gu J. Pathology of the thyroid in severe acute respiratory syndrome. Human Pathology 200738 95-102. (https://doi. org/10.1016/j.humpath.2006.06.011)

13 Spencer C, Eigen A, Shen D, Duda M, Qualls S, Weiss S \& Nicoloff J. Specificity of sensitive assays of thyrotropin (TSH) used to screen for thyroid disease in hospitalized patients. Clinical Chemistry 198733 1391-1396. (https://doi.org/10.1093/clinchem/33.8.1391)

14 Rosenfarb J, Sforza N, Rujelman R, Morosan Allo Y, Parisi C, Blanc E, Frigerio C, Fossati P, Caruso D, Faingold C et al. Relevance of TSH evaluation in elderly in-patients with non-thyroidal illness. Journal of Endocrinological Investigation 201942 667-671. (https://doi. org/10.1007/s40618-018-0967-0)

15 Bashkin A, Yaakobi E, Nodelman M \& Ronen O. Is routine measurement of TSH in hospitalized patients necessary? Endocrine Connections 20187 567-572. (https://doi.org/10.1530/EC-18-0004)

16 Wartofsky L \& Burman KD. Alterations in thyroid function in patients with systemic illness: the 'euthyroid sick syndrome'. Endocrine Reviews 19823 164-217. (https://doi.org/10.1210/edrv-3-2164)

17 Carle A, Andersen SL, Boelaert K \& Laurberg P. MANAGEMENT OF ENDOCRINE DISEASE: Subclinical thyrotoxicosis: prevalence, causes and choice of therapy. European Journal of Endocrinology 2017176 R325-R337. (https://doi.org/10.1530/EJE-16-0276)

18 Scappaticcio L, Trimboli P, Keller F, Imperiali M, Piccardo A \& Giovanella L. Diagnostic testing for Graves' or non-Graves' hyperthyroidism: a comparison of two thyrotropin receptor antibody immunoassays with thyroid scintigraphy and ultrasonography. Clinical Endocrinology 202092 169-178. (https://doi.org/10.1111/ cen.14130)
19 Carella C, Mazziotti G, Amato G, Braverman LE \& Roti E. Clinical review 169: interferon-alpha-related thyroid disease: pathophysiological, epidemiological, and clinical aspects. Journal of Clinical Endocrinology and Metabolism 200489 3656-3661. (https:// doi.org/10.1210/jc.2004-0627)

20 Iyer PC, Cabanillas ME, Waguespack SG, Hu MI, Thosani S, Lavis VR, Busaidy NL, Subudhi SK, Diab A \& Dadu R. Immune-related thyroiditis with immune checkpoint inhibitors. Thyroid 201828 1243-1251. (https://doi.org/10.1089/thy.2018.0116)

21 Yao XH, Li TY, He ZC, Ping YF, Liu HW, Yu SC, Mou HM, Wang LH, Zhang HR, Fu WJ et al. A pathological report of three COVID-19 cases by minimally invasive autopsies. Zhonghua Bing Li Xue Za Zhi 2020 49 E009.

22 Li MY, Li L, Zhang Y \& Wang XS. Expression of the SARS-CoV-2 cell receptor gene ACE2 in a wide variety of human tissues. Infectious Diseases of Poverty 20209 45. (https://doi.org/10.1186/s40249-02000662-x)

23 Hoffmann M, Kleine-Weber H, Schroeder S, Kruger N, Herrler T, Erichsen S, Schiergens TS, Herrler G, Wu NH, Nitsche A et al. SARSCoV-2 cell entry depends on ACE2 and TMPRSS 2 and is blocked by a clinically proven protease inhibitor. Cell 2020181 271.e8-280.e8. (https://doi.org/10.1016/j.cell.2020.02.052)

24 Lodigiani C, Iapichino G, Carenzo L, Cecconi M, Ferrazzi P, Sebastian T, Kucher N, Studt JD, Sacco C, Alexia B et al. Venous and arterial thromboembolic complications in COVID-19 patients admitted to an academic hospital in Milan, Italy. Thrombosis Research 2020191 9-14. (https://doi.org/10.1016/j.thromres.2020.04.024)

25 Russell CD, Millar JE \& Baillie JK. Clinical evidence does not support corticosteroid treatment for 2019-nCoV lung injury. Lancet 2020395 473-475. (https://doi.org/10.1016/S0140-6736(20)30317-2)

26 Dekkers OM, Horvath-Puho E, Cannegieter SC, Vandenbroucke JP, Sorensen HT \& Jorgensen JO. Acute cardiovascular events and all-cause mortality in patients with hyperthyroidism: a populationbased cohort study. European Journal of Endocrinology 2017176 1-9. (https://doi.org/10.1530/EJE-16-0576)

27 Inciardi RM, Lupi L, Zaccone G, Italia L, Raffo M, Tomasoni D, Cani DS, Cerini M, Farina D, Gavazi E et al. Cardiac involvement in a patient with coronavirus disease 2019 (COVID-19). JAMA Cardiology 2020. (https://doi.org/10.1001/jamacardio.2020.1096)

28 Madjid M, Safavi-Naeini P, Solomon SD \& Vardeny O. Potential effects of coronaviruses on the cardiovascular system: a review. JAMA Cardiology 2020 In pr. (https://doi.org/10.1001/ jamacardio.2020.1286)

29 Cho J \& Afolabi B. Ventricular fibrillation associated with Graves' disease and amiodarone induced thyrotoxicosis. Cardiovascular Endocrinology and Metabolism 20198 119-124. (https://doi. org/10.1097/XCE.0000000000000184)

30 Koulouri O, Moran C, Halsall D, Chatterjee K \& Gurnell M. Pitfalls in the measurement and interpretation of thyroid function tests. Best Practice and Research: Clinical Endocrinology and Metabolism 201327 745-762. (https://doi.org/10.1016/j.beem.2013.10.003)

31 Mehta P, McAuley DF, Brown M, Sanchez E, Tattersall RS, Manson JJ $\&$ HLH Across Speciality Collaboration, UK. COVID-19: consider cytokine storm syndromes and immunosuppression. Lancet 2020 395 1033-1034. (https://doi.org/10.1016/S0140-6736(20)30628-0)

32 Minciullo PL, Ruggeri RM, Vita G, Benvenga S \& Gangemi S. Development of Hashimoto's thyroiditis after subacute thyroiditis: an unusual patient. Thyroid 200919 73-74. (https://doi.org/10.1089/ thy.2008.0234)

Received 8 April 2020

Revised version received 15 June 2020

Accepted 6 July 2020 